\title{
Mitral Valve Abnormality Detection by fully end to end with Deep Neural Network
}

\author{
Vishal Chandra, Vinay Singh, Prattay Guha Sarkar
}

\begin{abstract}
The purpose of this research is to automatically identify normal and abnormal mitral leaflets in an apical four-chamber view.one of the widely spread valvular diseases is mitral valve disease in underdeveloped countries, still a burden for health sociality as well as countries. around 80 percent of valvular diseases are mitral valve disease problems. As far as World Heart Foundation Guidelines are concerned, It is totally based on mitral leaflets morphology. Due to the dependency on the sonographer's experience, it is highly subjective for argument. Measurement of thickness of leaflets, calcification detection, the pliability of leaflets required high experience about echocardiography as well as morphology. The motive of this research is to automatically identify the normal or abnormal mitral valve. If there is an abnormality in mitral leaflets then further investigation needed otherwise there is no further investigation that means measuring thickness, mitral valve area should not be required to measure. This research consists of two parts first automatically localize the region of interest second classifies the mitral leaflets whether normal or abnormal for localization yolo3 model mechanism with custom backend instead of darknet is used for taking area of interest automatically and for classification of normal and abnormal mitral leaflets, proposed pipeline is used, having f1, mAp score, and other matrices have measured.PR and ROC curves are drowned to support the results in the evaluation. the motive of this research is to serve nonexpert to identify abnormalities in mitral leaflets and sonographers to assess more efficiently. We used the Apical four-chamber view for this research.
\end{abstract}

Keywords: Object detection, Mitral valve detection, classification, abnormalities.

\section{INTRODUCTION}

99 percent of cases of abnormal mitral leaflets are RHD (Rheumatic Hear Dieses). In developing countries, Rheumatic heart disease (RHD) is due to Acute rheumatic fever, having the main cause of autoimmune response to group A streptococcal pharyngitis infection. since using hygiene food and availability of modern antibiotics treatment of such disease, there are very few cases of RHD observed, but RHD is still a big burden in the countries who are under development or undeveloped countries, it is mainly

Revised Manuscript Received on February 05, 2020.

* Correspondence Author

Vishal Chandra*, computing and information technology Usha Martin University, Ranchi, India. Email: vcvishalchandra@gmail.com.

Vinay Singh, computing and information technology Usha Martin University, Ranchi, India, Email: vinaysinghuma@gmail.com

Prattay Guha Sarkar, cardiology, RIMS, Ranchi, India. Email: prattay_2904@yahoo.com.

(C) The Authors. Published by Blue Eyes Intelligence Engineering and Sciences Publication (BEIESP). This is an open access article under the CC BY-NC-ND license (http://creativecommons.org/licenses/by-nc-nd/4.0/) responsible for cardiovascular morbidity and mortality in patients [1]. The outcome RHD is Mitral mixed lesions, mitral regurgitation (MR) and Mitral Stenosis (MS), which leads to heart failure. RHD can be accurately and easily clinically detected or diagnosed only by echocardiography. In the previous 25-years RHD spawn over globally [2], $34 \times 10^{6}$ patients of RHD, have lost of $9.5 \times 10^{6}$ life years and 323500 death were estimated alone in 2015 due to it. Although estimations were fallen significantly due to data unavailability of very fewer regions around the globe, misdiagnosis and not inclusion of subclinical RHD in the study lead to death. These aspects must not be overlooked due to facts coming out from studies [3], every 3 out of 10 clinical RHD cases exists sub-clinical disease and it cannot be ruled out. in the early stage of RHD treatment is possible without compromising much with health situations. Availability of portable echocardiography system, drastically increase in the rate of identification RHD WHF (World Heart Federation) has proposed guidelines for the assessment of RHD through echocardiography modality [4]. RHD It mostly affects heart muscles, the heart's valves, mostly its leaflets. Assessment of RHD through WHF guidelines for echocardiography based upon functional and morphological ways for mitral leaflets. Brightness mode (B mode) is used for identifying the mobility and morphology of mitral leaflets in echocardiography view of mitral valve mobility and morphology are assessed using brightness mode (B Mode) of the apical four-chamber view (A4C) views abnormal and normal leaflets are shown in Fig. 1a and 1b.

Due to higher inter-observer variability assessment of the Mitral valve is performed by studying AMVL (Anterior Mitral Valve Leaflet) instead of PMVL (Posterior Mitral Valve Leaflet [4]. Studies of mitral valve recommend that the most affected part of mitral leaflets is of its tip [5]. In underdeveloping and undeveloped country assessment, a higher operator's experience and a lot of time required which is very difficult to manage, the prime goal of this research to minimize the dependency upon operators in screening settings, and also to reduce subjectivity, for the improvement of diagnosis. Echocardiographic images are highly affected by signal dropout speckles, shadows, and attenuation. Images are also affected by the correct gain setting and individual operator which may lead to the wrong diagnosis. The authors proposed a pipeline to locate mitral leaflets in an apical four-chamber view with classifying them. Mitral leaflets which are useful to determine abnormalities rheumatic, congenital, degenerative leaflets in figure2.

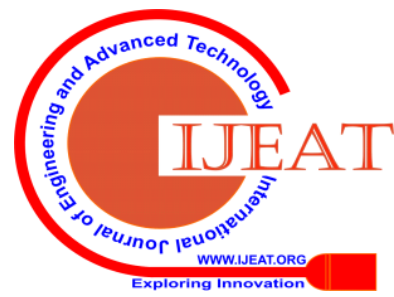




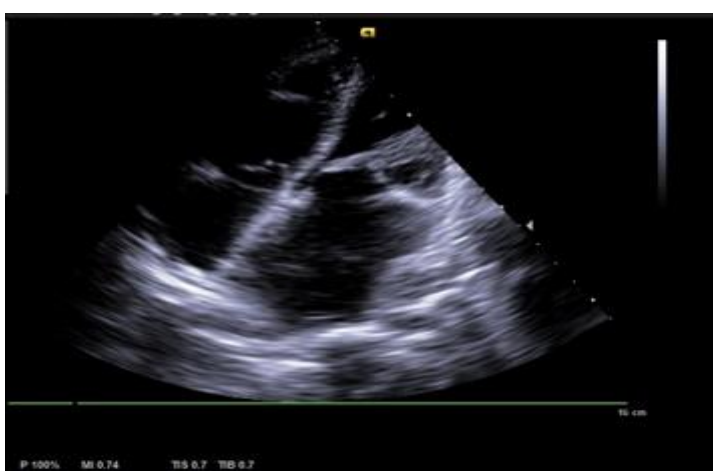

Figure 1a

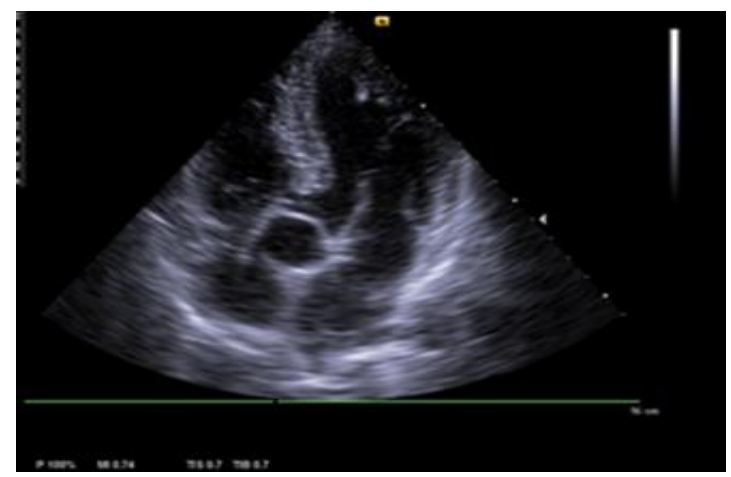

Figure 1b

\section{LITERATURE SURVEY}

In the past four years various researches on object detection. localization and classification of objects in either space, time or both have done. [9] For the identification of a landmark on the distal femur surface by using /CNNs2D MRI slices are used.[10] They did reformulate the problem to localized the regions of interest (ROIs) around anatomical regions (descending aorta, aortic arch, and heart) using 3D CT volume by results with 3D rectangular bounding box. [11] authors used pre-trained CNNs with RBM to reformulate the problem. [12] used CNNs for landmark locations, for it they used directly regression and used mapping, each landmark, ground truth data and for training and prediction by network landmark maps are performed by Gaussian representation. For the detection of landmark, the authors used reinforcement learning [13] for reducing the complexity of the model [14] used the method that decomposed 3D bleeding in the brain.[18] used 3D CNN. [19] used the learning method of weakly supervise for the detection of nodules in lesions in mammography and chest radiography. [20] used CNNs for the detection of thyroid papillary cancer in thyroid ultrasound to detect thyroid papillary cancer. Reformulation of the same problem done by [21]. [22] authors used inceptionv3 for the detection of thyroid nodules by using the Inceptionv3 model in ultrasound.[23] compare different models for the detection of regional wall motion abnormalities. [24] authors used 3D CNNs for the classification of ejection fraction. [25] they used CNNs for different echo view classification.

\section{DATA COLLECTION AND ANNOTATIONS}

Data is collected from cosy care hospital, Ranchi, Jharkhand, India under informed consent of all subjects. A total of 30 videos is taken from 30 different subjects having 10 rheumatics and 20 non-rheumatics with age from 15-70 years. Total 400 images obtained by taking during the beginning of systole, mid systole and end-systole of the left ventricle to capture mitral leaflets thus 400 images chosen for training and 30 for testing having 15 rheumatic and 15 non-rheumatics with 623 images. Data annotation is done by experienced sonographers using labelimg [26] tool which is freely available on GitHub. Interobserver variability is not significant by observing the length of leaflets and their opening width. classification of mitral leaflets ais also categorized by an experienced sonographer.

\section{YOLO3}

Authors named it "You only look once", faster among another object detection model like SSD, RetinaNet. Although it is not the most accurate algorithm for object detection but due to less inference time it is one of the best choices to select without losing much accuracy. It is better, stronger but not faster than yolo2 due to backend DarkNet53, which makes it more complex but makes more accurate. The problem with yolo2 was unable to detect smaller objects, which overcome by yolo3. Backend used by yolo3 is DarkNet53 figure 3, which has a 53-layer network, which trained on Imagenet. For the better in detection, 53 layers were added into DarkNet19, which results in 106-layer FCN architecture for YOLO v3. Figure 2 shows the architecture of the yolo3 CNN model which is like FPN architecture.

Figure 3 shows yolo3 detect an object on three different scales it is its salient features. YOLO is an FCN and its output is $1 \mathrm{x} 1$ kernel of the feature map. In YOLO v3, for the detection process, $1 \times 1$ detection kernels on feature maps are used with three different sizes and three different places in the network.

Fundamentally in Yolo, the given image is partitioned into $\mathrm{S} \times \mathrm{S}$ grid cells, every grid cell predicts one and only one object. In each grid cell,

convolution into three one-dimensional CNN for the detection bifurcation of the carotid artery. For the detection of the aortic valve in TEE, the authors used a method of deep learning called sparse adaptive [13]. For detection of 12 standard planes in mid-pregnancy [14] of fetal ultrasound used CNNs, applied Long short-term memory models. Detection of systolic and [15] diastolic movement in cin-MRI of heart. Authors integrated CT and PET and used multi-stream [16] CNN's for detection of internal

- $\mathrm{B}$ is the number of bounding boxes that can be predicted also provides a confidence score.

- only one object is detected for any number of predicted bounding boxes,

- each and every box conditional probability is provided.

DarkNet53 uses 18.57 billion parameters. Yolo3 does predictions at distinct scales.

1. The last layer is a feature map layer

2. Goes two-layer back upsamples by 2, after its higher resolution feature map is taken and element-wise addition is done with merged upsampled feature map after it applies convolutional filters on the merged map and makes the second prediction. 
3. Repeating the 2 procedures until the higher-level semantic structure of the feature map layer has achieved and object location with better spatial resolution information is achieved.

YOLOv3 uses 9 anchor boxes. For each scale, there are three. The structure of DarkNet is shown in figure 4 used for this research.

\section{PROPOSED MODEL}

Models consist of 19 having 8 convolutional layers, 4 MaxPooling.one Flatten, 2 dense and 2 dropout layers with 1 SoftMax layer figure 4.

Description of the proposed model

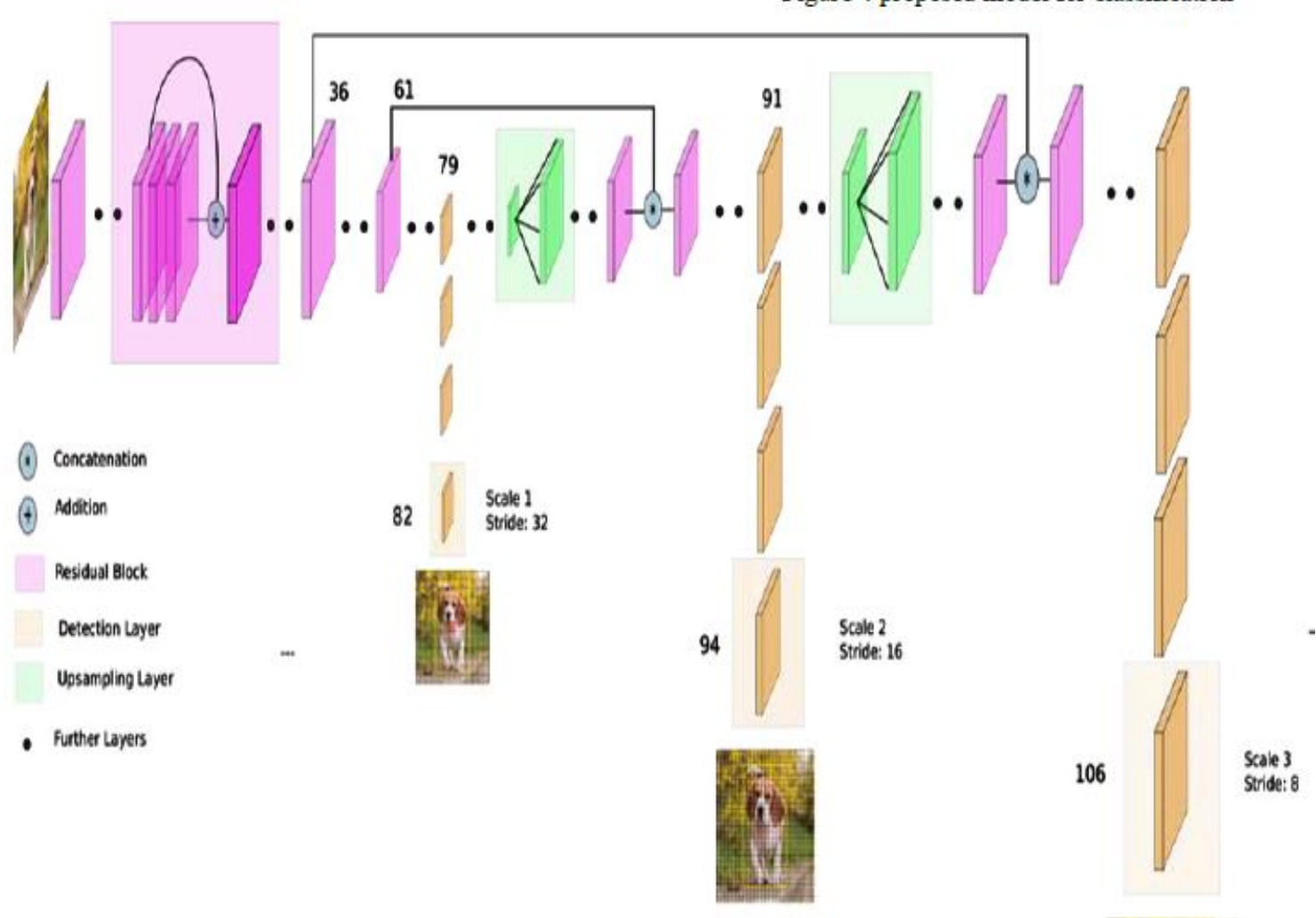

YOLO V3 network Architecture

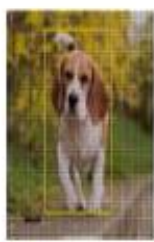

Figure 3. Image credits https://towardsdatascience.com/yolo-v3-object-detection-53fb7d3bfe6b

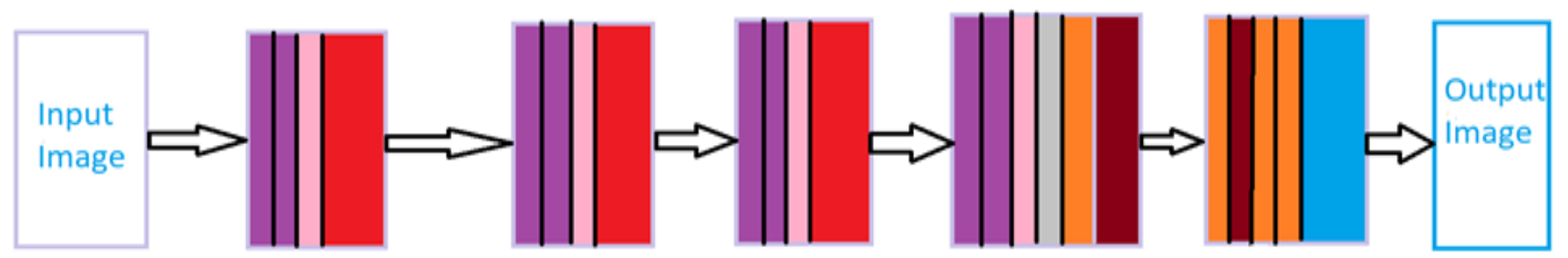

convolutional layer

Maxpooling layer

BatchNormalization

Dense Layer

Figure 4 proposed model for classification

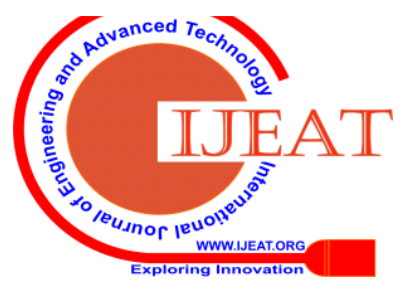


- First block convolutional layer $(16,3,3)$

- Second block convolutional layer $(32,3,3)$

- Second block convolutional layer $(64,3,3)$

- Second block convolutional layer $(128,3,3)$

- 2 Dense layers have 128

- And 2 Dropout is 0.5

- Activation function ReLU is used in each convolutional layer

- In the end, the sigmoid layer is applied

\section{PROPOSED WORK}

our whole work is divided into two parts first automatic detection of the mitral valve region which is our region of interest by yolo3, it gives mitral leaflets bounded area, the second part is to use the proposed model to identify or classify mitral leaflets whether they are normal or abnormal. The whole system works automatically end to end, no manual or human intervention required. The whole pipeline is shown in figure 5.

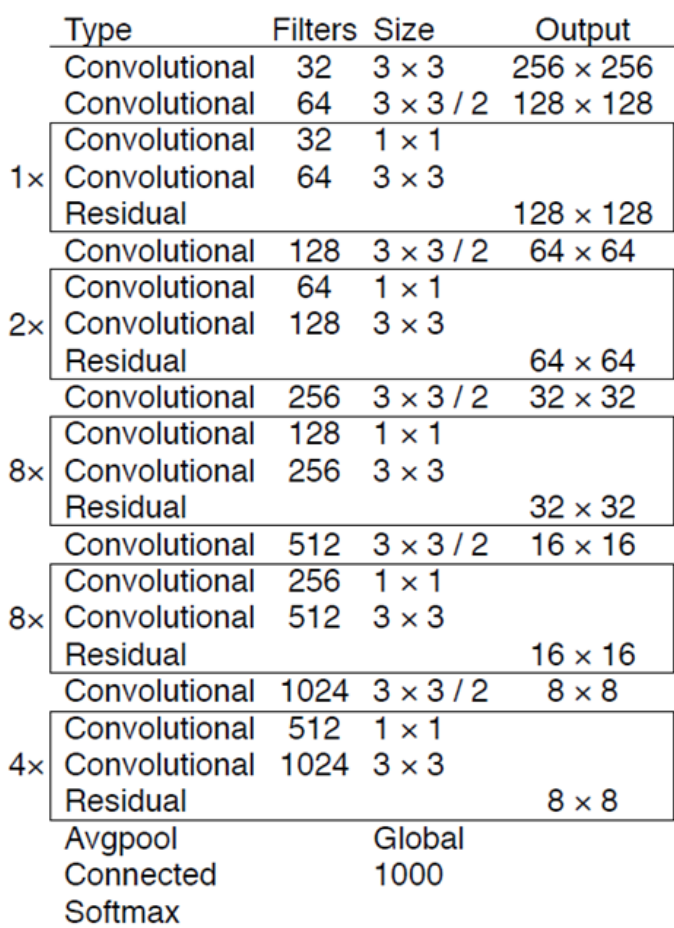

Figure 2 DarkNet53
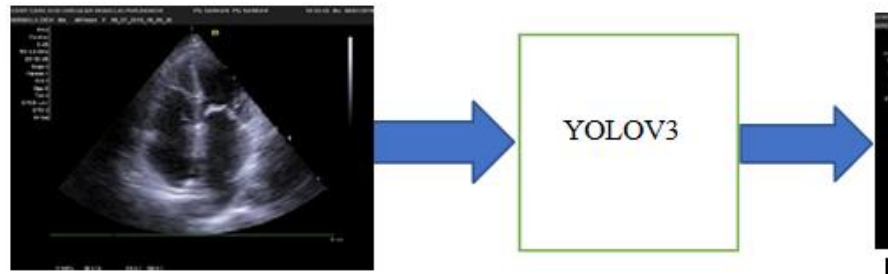

Abnormal Leaflet

Normal Leaflet
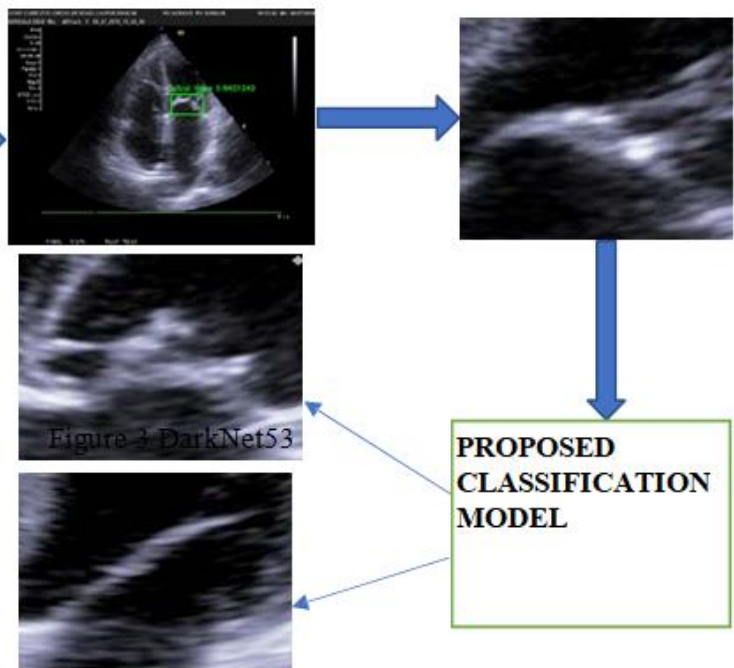

PROPOSED

CLASSIFICATION MODEL

Figure 5 Proposed pipeline.

Table 1. yolo3 configuration

\begin{tabular}{|c|c|}
\hline $\begin{array}{c}\text { Total Number of } \\
\text { parameters }\end{array}$ & 18.57 billion \\
\hline Image size & $512 \times 512$ \\
\hline Anchors & $\begin{array}{c}45,22,46,30,52,44,57,56, \\
57,34,59,98] \text { using k-Mean } \\
\text { clustering Algorithm }\end{array}$ \\
\hline $\begin{array}{c}\text { Maximum box per } \\
\text { image }\end{array}$ & 9 \\
\hline Batch size & 2 \\
\hline Learning rate & $1 \mathrm{e}-4$ \\
\hline Number of epochs & 40 \\
\hline Number of classes & 1 Mitral Valve) \\
\hline Validation Matrices & mAP \\
\hline
\end{tabular}

Retrieval Number: C5372029320/2020@BEIESP
Table 2. Configuration of the proposed classification model

\begin{tabular}{|c|c|}
\hline $\begin{array}{c}\text { Total Number of } \\
\text { parameters }\end{array}$ & $8,748,433$ \\
\hline Image size & $128 \times 128$ \\
\hline Batch size & 5 \\
\hline Learning rate & $1 \mathrm{e}-4$ \\
\hline Number of epochs & 20 \\
\hline Validation Matrices & \\
\hline
\end{tabular}


Yolov3 is trained by the collected dataset took $8 \mathrm{hr}$ for training, its configurational parameters are shown in table 1. The trained model is used to apply on test datasets to create a bounding box in which mitral leaflets are clearly visible. The output of yolov3 is cropped to the bounding box for the input of the classifier model. The cropped image is given as an input to the classification model which gives whether the image of normal and abnormal mitral leaflets. Detection layer is $\mathrm{DL}=1 \times 1 \times(B \times(5+C)$

$\mathrm{B}=9$ (number of bounding boxes)

$\mathrm{C}=1$ (Mitral Valve)

The configurational of the classification model is shown in table 2. For training of the proposed moded took only $1 \mathrm{hr}$ with 20 epochs.

\section{RESULTS}

After total training time 8hr 20min model trained without overfitting. Yolov3 has given $85 \mathrm{mAP}$ which is higher. $\mathrm{PR}$ (Precision-Recall) curve on testing data shown in figure 6 shows the performance of yolov3 to localize mitral leaflets inside bounding boxes. The performance summary is shown in table 3. As far as the proposed classification model is concerned classification accuracy reached 98.8 percent on test data that never been used in training having F1 score 0 . 99, testing accuracy 98.5 misclass 0.0144 , ROC curve with AUC 99, confusion matrix have drowned, shown in Figures 7 and 8 respectively. The output of classification results shown in figure 9. summary of the classification model is shown in table 4 .

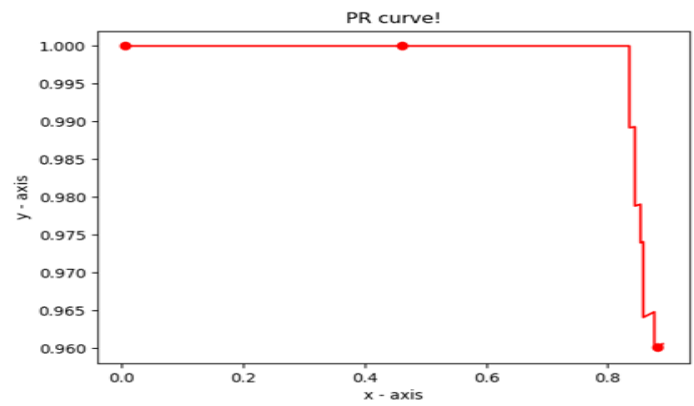

Figure 6 PR Curve of Mitral Valve

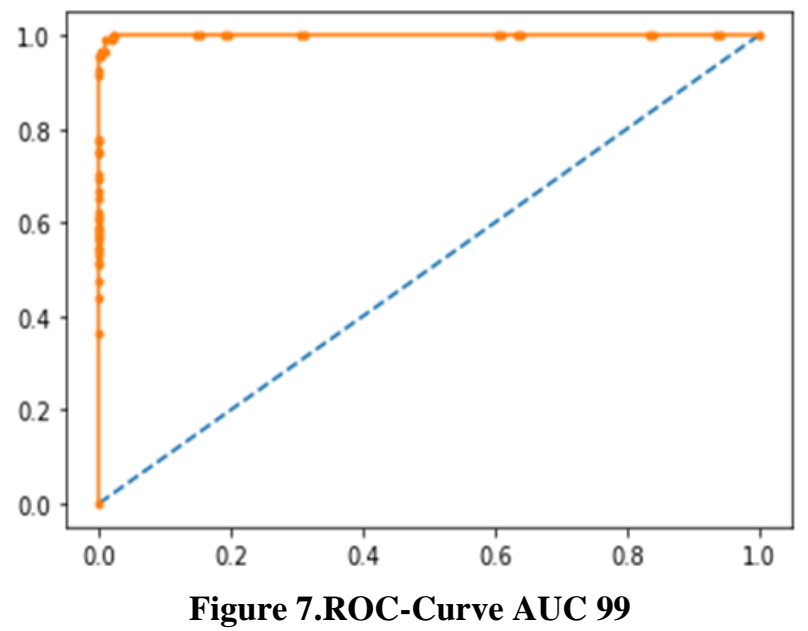

Figure 7.ROC-Curve AUC 99

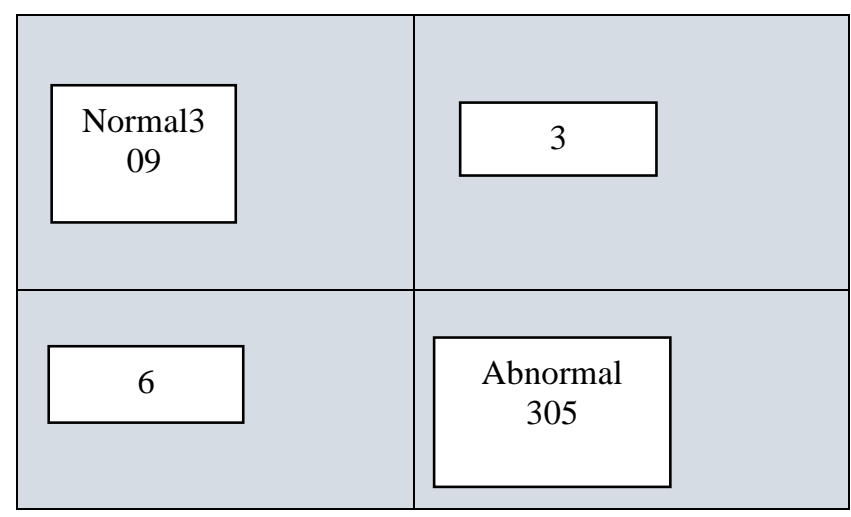

Figure 8 confusion Matrix

Classification report

\begin{tabular}{|c|c|c|c|}
\hline 0 & 0.98 & 0.99 & 0.99 \\
\hline 1 & 0.99 & 0.98 & 0.99 \\
\hline \multicolumn{3}{|l|}{ accul } & 0.99 \\
\hline
\end{tabular}

$\begin{array}{ccccc}\text { macro avg } & 0.99 & 0.99 & 0.99 & 623 \\ \text { weighted avg } & 0.99 & 0.99 & 0.99 & 623\end{array}$

\section{CONCLUSION}

Based on this research it has observed that deep learning methods can assist accurately to some extent where novice sonographers make mistakes. It is a stepping stone in the pathway of deep learning research in echocardiography.it will help sonographers to identify automatically abnormalities inn mitral leaflets which are by any case congenital, rheumatic, vegetation.in spite of the small dataset for training, the proposed method gives promising results.

\section{REFERENCES}

1. S. J. S. B and S. P. Awate, "Hierarchical Generative Modeling and Monte-Carlo EM in Riemannian Shape," Miccai 2016, vol. 2, pp. 191-200, 2016.

2. A. Barbu, L. Lu, H. Roth, A. Seff, and R. M. Summers, "An analysis of robust cost functions for CNN in computer-aided diagnosis," Comput. Methods Biomech. Biomed. Eng. Imaging Vis., vol. 6, no. 3, pp. 253-258, 2018.

3. S. Bauer, N. Carion, P. Schüffler, T. Fuchs, P. Wild, and J. M. Buhmann, "Multi-Organ Cancer Classification and Survival Analysis," no. Nips, pp. 1-5, 2016.

4. M. R. Berg, "Ingen gevinst av str??lebehandling ved livmorkreft," Tidsskr. den Nor. Laegeforening, vol. 133, no. 23-24, p. 2459, 2013.

5. R. Cervera, "Recent advances in antiphospholipid antibody-related valvulopathies," J. Autoimmun., vol. 15, no. 2, pp. 123-125, 2000.

6. B. D. de Vos, J. M. Wolterink, P. A. de Jong, M. A. Viergever, and I. Išgum, "2D image classification for $3 \mathrm{D}$ anatomy localization: employing deep convolutional neural networks," Med. Imaging 2016 Image Process., vol. 9784, p. 97841Y, 2016.

7. A. G. Howard et al., "MobileNets: Efficient Convolutional Neural Networks for Mobile Vision Applications," 2017.

8. S. Hwang and H. E. Kim, "Self-transfer learning for weakly supervised lesion localization,” Lect. Notes Comput. Sci. (including Subser. Lect. Notes Artif. Intell. Lect. Notes Bioinformatics), vol. 9901 LNCS, pp. 239-246, 2016.

9. ] E. Marijon, D. S. Celermajer, and X. Jouven, "Rheumatic heart disease — An iceberg in tropical waters,” N. Engl. J. Med., vol. 377, no. 8, pp. 780-781, 2017.

10.N. Navab, J. Hornegger, W. M. Wells, A. F. Frangi, and D. Hutchison, "Medical Image Computing and Computer-Assisted Intervention," pp 507-514, 2014. 
11.J. Redmon and A. Farhadi, "YOLO9000: Better, faster, stronger," Proc. 30th IEEE Conf. Comput. Vis. Pattern Recognition, CVPR 2017, vol. 2017-Janua, pp. 6517-6525, 2017.

12.J. Redmon and A. Farhadi, "YOLOv3: An Incremental Improvement," 2018.

13.B. Reméanyi et al., "World Heart Federation criteria for echocardiographic diagnosis of rheumatic heart disease-an evidence-based guideline," Nat. Rev. Cardiol., vol. 9, no. 5, pp. 297-309, 2012.

14.A. Teramoto, H. Fujita, O. Yamamuro, and T. Tamaki, "Automated detection of pulmonary nodules in PET/CT images: Ensemble false-positive reduction using a convolutional neural network technique," Med. Phys., vol. 43, no. 6, pp. 2821-2827, 2016.

15.M. J. J. P. Van Grinsven, B. Van Ginneken, C. B. Hoyng, T. Theelen, and C. I. Sánchez, "Fast Convolutional Neural Network Training Using Selective Data Sampling: Application to Hemorrhage Detection in Color Fundus Images," IEEE Trans. Med. Imaging, vol. 35, no. 5, pp. 1273-1284, 2016.

16.D. A. Watkins et al., "Global, regional, \& national burden of rheumatic heart disease, 1990-2015," N. Engl. J. Med., vol. 377, no. 8, pp. 713-722, 2017.

17.J. M. Wolterink, T. Leiner, B. D. de Vos, R. W. van Hamersvelt, M. A. Viergever, and I. Išgum, "Automatic coronary artery calcium scoring in cardiac CT angiography using paired convolutional neural networks," Med. Image Anal., vol. 34, pp. 123-136, 2016.

18.D. Yang, S. Zhang, Z. Yan, C. Tan, K. Li, and D. Metaxas, "AUTOMATED ANATOMICAL LANDMARK DETECTION ON DISTAL FEMUR SURFACE USING CONVOLUTIONAL NEURAL NETWORK Department of Computer Science, University of North Carolina at Charlotte , NC , US Department of Industrial and Systems Engineering , Rutgers University ,"pp. 17-21, 2015.

19.J. Yao, S. Wang, X. Zhu, and J. H. B, "Imaging Biomarker Discovery for Lung Cancer," Miccai 2016, vol. 1, pp. 649-657, 2016.

20.H. Li et al., "An improved deep learning approach for detection of thyroid papillary cancer in ultrasound images," Sci. Rep., vol. 8, no. 1, pp. 1-12, 2018.

21.L. Ma, C. Ma, Y. Liu, and X. Wang, "Thyroid diagnosis from SPECT images using convolutional neural network with optimization," Comput. Intell. Neurosci., vol. 2019, 2019.

22. J. Song et al., "Ultrasound image analysis using deep learning algorithm

23.diagnosis of thyroid nodules," Medicine (Baltimore)., vol. 98, no. 15, p e15133, 2019.

24.Kusunose K, Abe T, Haga A, Fukuda D, Yamada H, Harada M, et al. A Deep Learning Approach for Assessment of Regional Wall Motion Abnormality From Echocardiographic Images. JACC Cardiovasc Imaging 2019. https://doi.org/10.1016/j.jcmg.2019.02.024.

25.Silva JF, Silva JM, Guerra A, Matos S, Costa C. Ejection Fraction Classification in Transthoracic Echocardiography Using a Deep Learning Approach. Proc - IEEE Symp Comput Med Syst 2018;2018-June:123-8. https://doi.org/10.1109/CBMS.2018.00029.

26.Madani A, Arnaout R, Mofrad M, Arnaout R. Fast and accurate view classification of echocardiograms using deep learning. Npj Digit Med 2018;1:1-8. https://doi.org/10.1038/s41746-017-0013-1.

27. https://github.com/tzutalin/labelImg. 


\section{Results}
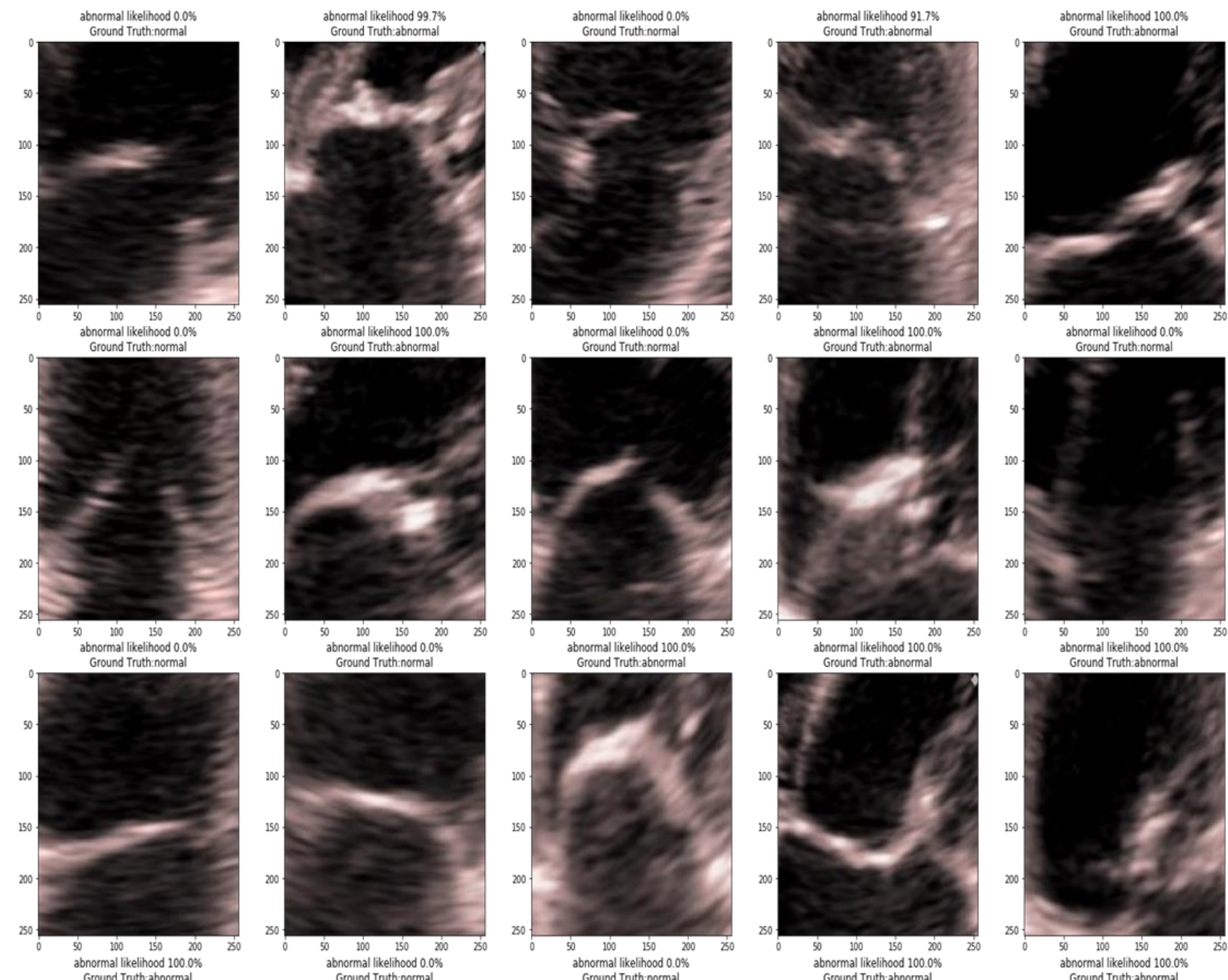

abnormal likelihood 100.03
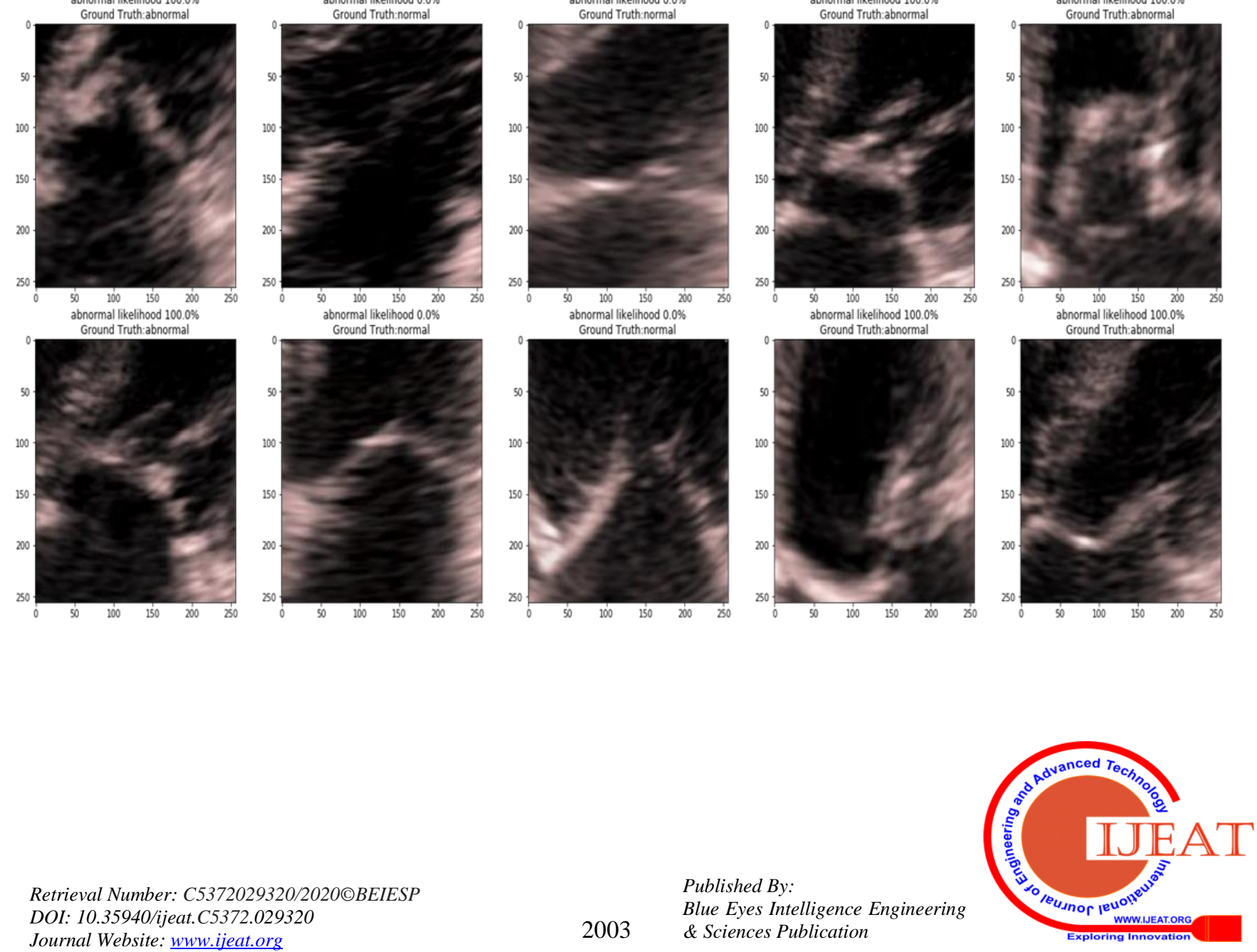


\section{AUTHORS PROFILE}

Mr. Vishal Chandra is a PhD Scholar at Usha Martin University, Ranchi, Jharkhand. Research area is Artificial intelligence. He published 14 research paper in national and international journals, also presented 4 conference papers.

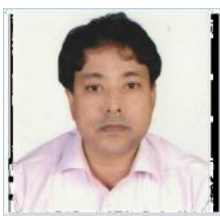

Dr. Vinay Singh received his Ph.D degree

from BITs Mesra, Ranchi, and Master of Technology in Computer science and Engineering from BITs Mesra, Ranchi, India. Presently he is working as an Associate Professor in Dept of Computer science and

Engineering in Usha Martin University, Ranchi. He is also empanelled with Wipro Technologies as a corporate trainer. He has published more than 20 papers in the International Journal and Conference. His Research area is Software Metrics and Quality, Machine Learning and Big Data.

Author-3

Photo
Dr.Prattay Guha Sarkar is MD (internal medicine), DM (cardiology), presently working as Assistant Professor of Cardiology at RIMS Ranchi. His research area includes cardiac interventions and imaging. 